\title{
Emergence of Traffic Lights Synchronization
}

\author{
Denise de Oliveira and Ana L. C. Bazzan \\ Instituto de Informática, UFRGS \\ C.P. 15064, 91501-970, P.Alegre, RS, Brazil \\ \{edenise,bazzan\}@inf.ufrgs.br
}

\begin{abstract}
Keywords-Agent-based simulation, Traffic lights Control, Swarm Intelligence

Abstract-

In control of urban traffic systems, synchronization of traffic lights is one of the measures used to improve the throughput, as it allows vehicles to pass an arterial without stop. Classical approaches are off-line, top-down planned and centralized. Moreover, they are inflexible since they cannot cope with dynamic changes in the traffic flow or depend too much on communication. This paper describes a bottom-up simulation approach where each traffic light is an agent and behaves like a social insect. Our approach is simulated using a microscopic model, with a scenario adapted from a city in Brazil. The volume of vehicles in an arterial and its vicinity was simulated under different situations: without any coordination between traffic lights, with fixed coordination, and with the approach proposed here, which is more flexible: traffic lights adapt to the current volume of vehicles by selecting the appropriate signal plan. Besides, it considers not only the traffic in the arterial, but also in secondary streets.
\end{abstract}

\section{Introduction AND Motivation}

Approaches to reduce traffic jams have been proposed in several disciplines like transportation engineering, physics, and artificial intelligence, among others. A classical one is to coordinate or synchronize traffic lights so that vehicles can traverse an arterial in one traffic direction, with a specific speed, without stopping. Thus, synchronization here means that if appropriate signal plans are selected to run at the adjacent traffic lights, a "green wave" is built so that drivers do not have to stop at junctions. There are several reasons why this approach may fail.

In traffic networks without well-defined traffic flow patterns like for instance morning flow towards downtown and it similar afternoon rush hour, that approach may not be effective. This is clearly the case in big cities where the business centers are no longer located exclusively downtown. Also, in some cities, "secondary" streets might be as important as main arterials due to the saturation of these. Finally, a priori determination of the appropriate signal plans for the different times of a day is a complex task that requires a lot of knowledge about dynamic traffic flow.

These issues show that simple offline optimization or even on-line optimization of the synchronization in one arterial alone cannot cope with changing traffic patterns. This happens because traffic is a highly dynamic process, thus the currently optimal signal plan can hardly be determined in advance. With an increasing volume of traffic, this situation becomes more and more unacceptable. Thus, flexible and robust approaches are not only attractive, but necessary.

Authors partially supported by CAPES and CNPq

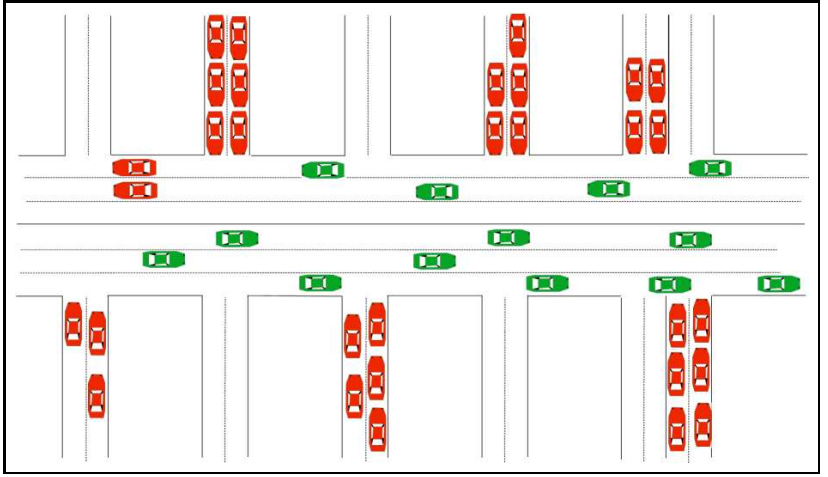

Fig. 1. Example of a green wave causing a traffic jam in secondary streets

Figure 1, where the red cars are stopped and the green ones are in movement, shows an example of the inefficiency of a green wave computed offline, based on historical data. In the particular moment depicted, the main arterial is not so jammed as to require high priority. On the other hand, "secondary" streets crossing the arterial are jammed. Our approach seeks to replace the traditional arterial green wave by "shorter green waves" in segments of the network. Of course in some key junctions conflicts may appear because in almost all practical situations, a signal plan do not allow synchronization in more than one traffic direction.

Bottom-up approaches offer more flexible solutions, as they are able to adapt to changing patterns in traffic, and allow the study of the emergence of groups of coordinated traffic lights. This is not the practice in transportation engineering: currently the synchronization is planned offline by the traffic engineer, or it is computed by algorithms which take only the main arterial in consideration, changing only the direction of the synchronization.

This paper presents an approach in which each junction (plus its traffic lights) is an agent and behaves like a social insect that grounds its decision-making on mass recruitment mechanisms found in social insects [3], [8]. Henceforth we use the terms crossing, junction, and traffic light indistinctly. This is so because in fact, in each crossing or junction only one signal plan runs in a set of traffic lights (despite the fact that one sees two or three of these) so that the set of traffic lights must be seen as a single entity.

Signal plans are seen as tasks to be performed by the insect. Thus, from a metaphor of the social insects, in our approach the ability of changing tasks in order to suit the colony needs is located in each crossing or junction. Stimuli to perform a task or, sometimes, to change tasks, are provided by the vehicles that, while waiting 
for their next green phase, continuously produce some "pheromone". Thus the volume of traffic coming from one direction can be evaluated by the agent, and this may trigger some signal plan switching. No other information is available for the intersection agents.

Our approach was realized on a microscopic traffic simulator. This is necessary in any agent-based approach since it is desirable that the objects of the simulation are modeled at individual level. Thus, the next section presents some basic concepts about traffic simulation regarding synchronization of traffic lights and the simulator. Section III then discusses the agent-based model, while Section IV presents the scenario we simulated as well as the results of these simulations. Section V summarises the contributions and discusses future extensions.

\section{Traffic Signal Coordination and Simulation}

\section{A. Approaches}

Traffic Network Study Tool (TRANSYT) [10] is one wellknown algorithm for traffic light synchronization. It runs off-line and aims at optimizing the bandwidth of an arterial via the design of phases and offsets from one intersection to the adjacent one.

Similar tools are SCATS and SCOOT. However these are both based on online traffic volume information coming from loop-induced detectors installed in the roads. SCATS (Sydney Coordinated Adaptive Traffic System) [6] was initially developed for the Sydney area. It is a real-time control system, based on a decentralized architecture. It optimizes the length of cycle time and offsets, and allows some phases to be skipped at times. SCOOT (Split Cycle and Offset Optimization Technique) [5] is a centralized traffic control system developed by the Transportation Road Research Laboratory (UK). SCOOT also optimizes cycle and offset, as well as saturation rate. Although both deal with real time data, their concept is still based on synchronization in one main path.

Agent-based systems offer more flexible solutions. In [1] an approach is described in which each traffic light is modeled as an agent. Each agent has pre-defined signal plans to coordinate with other agents in the neighborhood. Different signal plans can be chosen in order to coordinate in a given traffic direction or during a pre-defined period of the day. This approach uses techniques of evolutionary game theory: intersections in an arterial are modeled as individually-motivated agents or players taking part in a dynamic process in which, due to the reward, not only their own local goals but also a global one can be taken into account. Moreover, each agent possesses only information about their local traffic states.

The benefits of this approach are threefold. First, it is not necessary to have a central agent to determine the direction of the coordination. Second, agents can build subgroups of synchronization which meet their own local and current needs in terms of allowing vehicles to pass in one given direction. Third, it avoids communication between agents when they have to decide which direction to priorize, i.e. there is no explicit communication or negotiation.

However, payoff matrices (or at least the utilities and preferences of the agents) are required, i.e these figures have to be explicitly formalized by the designer of the system. This makes the approach time consuming when many different options of coordination are possible (for example all four traffic directions: south, north, east, and west) and/or the traffic network is complex (for instance, not only a main arterial has to be considered but also many transversal and parallel streets).

\section{B. Synchronization in Arterials: Basics}

Signalized intersections are controlled by signal-timing plans which are implemented at traffic signals. A signaltiming plan (henceforth signal plan for short) is a unique set of timing parameters comprising basically the cycle length (the length of time for the complete sequence of the phase changes), and the split (the division of the cycle length $C$ among the various movements or phases).

The criteria for obtaining the optimum signal timing is that it should lead to the minimum overall delay at the intersection. This is usually achieved by using simulation or optimization programs. Several plans are normally required for an intersection (or set of intersections in the case of a synchronized system) to deal with changes in traffic flow.

The goal of coordinated or synchronized systems is to synchronize the traffic signals along an arterial in order to allow vehicles, traveling at a given speed, to cross the arterial without stopping at red lights. Besides the parameters mentioned above, the synchronized plans also need an offset i.e. the time between the beginning of the green phase at two consecutive traffic signals (only when they are synchronized).

Well designed signal plans can achieve acceptable results in un-congested streets in one flow direction. However synchronization in two opposing directions of an arterial is difficult to achieve, if not impossible, in almost all practical situations. The difficulty is that the geometry of the arterial is fixed and with it the spacing between adjacent intersections. Only in very special cases the geometry allows progression in opposite directions. Synchronization in four directions is, for practical purposes, impossible. Therefore an agent at a junction must select which plan to carry out, in analogy to a task selection.

As a measure of effectiveness of such systems, one generally seeks to optimize a weighted combination of stops and delays, a measure of the density (vehicles/unit of length) in the road or network, or travel time. Here we use the latter.

\section{Microscopic Traffic Simulator}

We use the Nagel-Schreckenberg model [7] which is a microscopic model for traffic simulation originally based on cellular-automata (CA). In short, each road is divided in cells with a fixed length. This permits the representation of a road as an array where vehicles may be positioned on its positions. Each vehicle has a speed which is represented by the number of cells it may safely advance at each time step. The vehicle behavior is expressed by some rules that represent a special form of car following behavior. This 
simple, yet valid microscopic traffic model can be implemented in such an efficient way that it is good enough for real time simulation and control of traffic.

As for the network representation, each road is described as a composition of nodes - representing junctions - and edges. The expression edge refers to directed edges representing one direction of motion on a road, i.e. one road usually consists of two (oppositely directed) edges.

In the urban traffic scenario, more elements were added such as traffic lights and more complex types of intersections. Thus, the simulation tool we developed consists of different elements like lanes, edges, vehicles, sources and sink (of vehicles), traffic lights, sensors and detectors. The topological configuration and parameter for the simulation dynamics are stored in a XML file. Basically, the simulator checks the static and dynamic network data read from the XML file for consistency and initializes the scenario. During the simulation it receives and updates dynamic data like vehicle volume, handles the simulation output, and updates the vehicle motions, traffic light, and data for statistics.

More details can be obtained in [9]. Here, we focus on the traffic light since it is the main object for the coordination. Each signalized junction has an agent which is in charge of deciding which signal plan to run.

\section{Using Metaphors of TASK Allocation IN Colonies of Social Insects to Model traffic Light AgEnts}

\section{A. Task Allocation}

Bonabeau et al. [2] present a mathematical model that formalizes a hypothesis of how the division of labor may happen in colonies of social insects. Interactions among members of the colony and the individual perception of local needs result in a dynamic distribution of tasks. Their model describes the colony task distribution using the stimulus produced by tasks that need to be performed and an individual response threshold related to each task. Each individual insect has a response threshold for each task to be performed. That means, at individual level, each task has an associated stimulus, like for instance the perception of waste as a stimulus for cleaning behavior.

The levels of the stimulus increase if tasks are not performed, or not performed by enough individuals. An individual that perceives (e.g. after walking around randomly) a task stimulus higher than its associated threshold, has a higher probability to do this task (Eq. 3). This model also includes a simple way of reinforcement learning where individual thresholds decreases when performing some task and increases when not performing. This double reinforcement process leads to the emergence of specialized individuals.

These concepts are used in our approach in the following way: each agent (traffic light/crossing) has a social insect behavior. It has different tendencies to execute one of its signal plans (each signal plan is considered an available task), according to the environment stimulus and particular thresholds. Besides these individuals, this approach also considers that each vehicle leaves a pheromone trace that can be perceived by the agents at the junction. This metaphor is realistic since many junctions have loop in- duction sensors which detect the counting of vehicles (and sometimes speed).

\section{B. Computation of Stimulus}

The liberated pheromone dissipates in a pre defined rate in time and its intensity indicates how high is the the traffic volume in the street portion. The pheromone trail can be considered as a stigmergic communication among adjacent traffic lights. The increase of the accumulated pheromone in a certain direction can be seen by the insect as a change in a task selection executed by its neighbor.

Each particular task in the Bonabeau et al. model [2] has one associated stimulus. The intensity of this stimulus can be related to a pheromone concentration, a number of encounters between individuals performing the task, or any other quantitative cue sensed by individuals. The traffic light stimulus is the average of the accumulated pheromone of all lanes (incoming and outgoing).

The accumulated pheromone in a lane, $d_{l, t}$, is the pheromone trail accumulated in the lane $l$ at time $t$. While the vehicles are waiting for the green light they remain releasing pheromone so its amount increases. However the pheromone also dissipates at a rate $\beta$.

$$
d_{l, t}=\frac{\sum_{t=0}^{w} \beta^{-t}\left(d_{l, t}\right)}{\sum_{t=0}^{w} \beta^{-t}}
$$

where:

$w$ time-window size

$\beta$ pheromone dissipation rate for each lane $l$

The stimulus $s$ of the plan $j$ is based in a weighted sum of accumulated pheromone in each phase of this plan. Each phase has a time share $\left(\Delta_{k}=\right.$ time $_{\text {end }}-$ time $\left._{\text {begin }}\right) /$ time $\left._{\text {cycle }}\right)$, that indicates how much green time the plan allows to a phase. A higher time interval indicates a phase priority in the plan.

$$
s_{j}=\sum_{k=0}^{n}\left((1-\alpha) d_{{i n_{k, t}}}+\alpha d_{\text {out }_{k, t}}\right) \Delta_{k}
$$

where:

$n \quad$ number of phases of the signal plan $j$

$d_{i n_{k, t}}$ is the accumulated pheromone trail in the input lanes in phase $k$ at time $t$

$d_{\text {out }_{k, t}}$ is the accumulated pheromone trail in the output lanes in phase $k$ at time $t$

$\Delta_{k}$ is the time share of the phase $k$

$\alpha$ constant employed to set different priorities to the input and output lane densities

\section{Actual Plan Allocation}

Behavioral flexibility of changing plans is a consequence of environmentally induced changes in stimulus and threshold. Every signal plan possess associated stimuli according to the direction towards this signal plan is biased. Individuals may change task because high levels of stimulus related to a direction exceed their response threshold. Equation 3 defines the response function (the probability of chose the 
plan $j$ as a function of stimulus intensity $s_{j}$ ) of the individual $i$.

$$
T_{\theta_{i j}}\left(s_{j}\right)=\frac{s_{j}^{2}}{s_{j}^{2}+\theta_{i j}^{2}}
$$

where:

$\theta_{i j}$ is the response threshold for the individual $i$ for executing the task $j$.

$s_{j}$ is the stimulus associated with the task $j$.

\section{Reinforcement}

We use the specialization model, where the threshold is updated in a self reinforced way. Each individual in the model has one response threshold to each task. These thresholds are updated (increasing or decreasing) according to two different coefficients. The response threshold $\theta$ is expressed as units of intensity of stimulus. The response threshold $\theta_{i j}$ of an individual $i$ when performing task $j$ during time interval of duration $\Delta t$ is:

$$
\theta_{i j}=\theta_{i j}-\xi \Delta t_{i j}
$$

where:

$\xi$ learning coefficient

$\Delta t$ time interval

The response threshold $\theta_{i j}$ of the agent $i$ when not performing task $j$ during time interval of duration $\Delta t$ is:

$$
\theta_{i j}=\theta_{i j}+\rho \Delta t_{i j}
$$

where:

$\rho$ forgetting coefficient

According to Gordon [4], real ants are directly influenced by their success in performing a given task. Successful ants are motivated to remain performing a task and unsuccessful ants are motivated to stop performing the task and change to another one. We have extended the Bonabeau et al. [2] model in order to include a success function as the coefficient that describes learning and forgetting at the same time (when the $l$ is negative the agent is forgetting). Equation IV-B defines this extension.

$$
\theta_{i j}=\theta_{i j}-l \Delta t
$$

where:

$l$ is the learning/forgetting coefficient.

$\Delta t$ is a normalized discrete time interval.

The success degree of the individual is given by Equation 7 and Equation 8, where a higher standard deviation of accumulated pheromone $\sigma$ (Equation 9, where $n$ is the number of street sections) leads to a smaller degree of success.

$$
\begin{gathered}
l=1-2 \sigma \\
l=2 e^{(-5 \sigma)}-1
\end{gathered}
$$

$\sigma$ is the standard deviation of accumulated pheromone trail in the sections.

$$
\sigma=\sqrt{\frac{1}{n-1} \sum_{k=1}^{n}\left(d_{i}-\bar{d}\right)^{2}}
$$

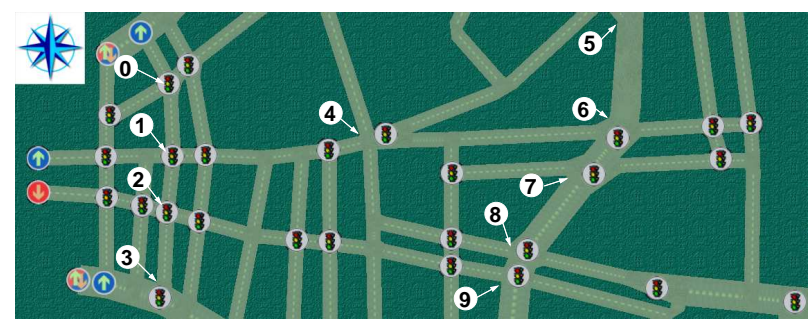

Fig. 2. Network and the traffic lights locations

where:

$n$ is the number of street sections.

$d_{k}$ accumulated pheromone in section $k$

$\bar{d}$ is the mean accumulated pheromone trail in $n$ sections.

The whole system tends to stay stable and suited to the traffic flow but can change in order to adapt to a new environment situation. Traffic lights in the same street with an intense traffic flow in a certain direction tend to adopt the synchronized plans and give priority for this direction.

\section{Description of the Scenario and Results of The Simulations}

\section{A. Scenario}

The scenario is part of a real network located in the city of Porto Alegre (Brazil). Figure 2 shows a simplified schema generated by a $3 \mathrm{D}$ visualization tool of our agentbased simulator. This set of streets was chosen due to the high traffic flow.

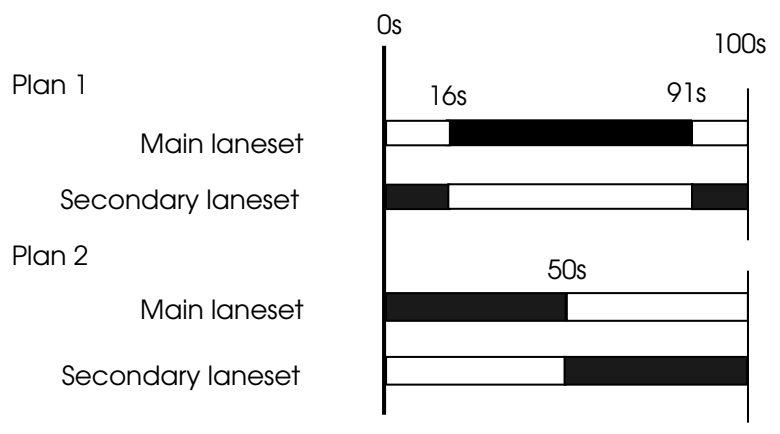

Fig. 3. Basic Signal Plans (the dark strip represents the green time)

The main street or arterial has 8 signalized junctions, each with two possible signal plans kinds: coordinated and non-coordinated. Besides, some other crossing roads also have signalized junctions. We focus our measurements also on two secondary streets: one is composed by crossings "0" to "3" (Figure 2) while the other is composed by crossings " 5 " to "9" Henceforth we refer to these as arterial, secondary 1, and secondary 2. In each arterial junction, signal plan 1 (SP1) gives priority to the main direction (west to east in the Arterial and North to South in the secondary streets) and it is synchronized with the adjacent traffic lights in this direction. Plan 2 is not synchronized with plans in neighboring junctions, and allocates equal share of green time for each direction, as we can see in Figure 3. Each traffic light can have more then two different 
signal plans according to its coordination possibilities. For instance, the traffic light located in node "1" has three different signal plans: one for coordinating to the neighbors in the West-East direction, one for coordinating with the neighbors in the North-south direction, while the last one is not coordinated in any direction.

Regarding coordinated or synchronized plans (e.g. SP1), the difference between this kind of plan running at two adjacent junctions is the offset. For instance, two adjacent junctions have the same basic synchronized plans but in the one upstream there is an offset of, say, 15 second which makes the green time start 15 seconds later than in the previous junction. This indicates that vehicles departing at one junction and traveling with the synchronization speed $V$ will be able to pass the next one 15 seconds later without stopping.

Vehicles are inserted in the network at sources located generally (but not only) at the borders of it. For instance, vehicles are inserted in the main street from a source located in the left side, where the blue edge appears (Fig. 2). This insertion happens with different rates in each street. We setup these rates according to real traffic flow information. Similarly, at the network borders, vehicles are removed from the scenario.

In the arterial, 36 vehicles/minute were inserted in its 3 lanes, defined by a 0.6 probability of a vehicle being inserted in each second of the simulation. Secondary 1 and 2 have an insertion rate of 24 vehicles/minute (the probability of a vehicle be inserted in each second is 0.4 ).

Besides, each junction has turning probabilities which can be computed from real data. Therefore, each vehicle either stays in the direction it is, or turns to another one.

Finally, the microsimulator allow us to define special vehicles called floating cars (FC) which have a fix route and collect data about the traffic conditions (e.g. regarding position and speed at each time step). Thus, we assigned three of these FCs to each one of the three routes of interest. In this simulation, all the vehicles (including the FCs) have a zero probability of deceleration, thus, if there is no jam, the vehicles tend to keep the maximum speed (in this case 4 cells/step or 12 meters/second).

\section{B. Results from Simulations}

The simulations presented in this section were generated using the simulator discussed in Section II-C with the scenario presented in Section IV-A.

In the beginning of the simulation, the network is empty and some time is needed for vehicles to reach all the portions of the network. Thus, in order to have a stable situation in the network, with a representative number of vehicle, we do not consider the initial time window.

The aim of the experiments is to compare the travel time taken by each FC during its assigned route, using both our approach and standard ones. Two situations are evaluated. In the first, there is no synchronization (i.e. all junctions run plans like SP2 in Figure 3). In the second case, we compare our approach to the situation in which junctions run synchronized plans but they are fix, i.e. the engineer decides how all traffic lights are synchronized in a fix way.
TABLE I: Average time to complete a route (seconds)

\begin{tabular}{l|l|l|l}
\hline Situation & \multirow{3}{*}{ Arterial } & $\begin{array}{l}\text { Recondary } \\
1\end{array}$ & $\begin{array}{l}\text { Secondary } \\
2\end{array}$ \\
\hline $\begin{array}{l}\text { No traffic } \\
\text { lights }\end{array}$ & 100.00 & 37.25 & 36.08 \\
\hline $\begin{array}{l}\text { Fixed } \\
\text { synchro- } \\
\text { nization }\end{array}$ & 161.29 & 287.70 & 255.67 \\
\hline $\begin{array}{l}\text { No syn- } \\
\text { chroniza- } \\
\text { tion }\end{array}$ & 205.16 & 130.19 & 287.46 \\
\hline $\begin{array}{l}\text { Swarm } \\
\text { with fix } \\
\text { threshold }\end{array}$ & 305.01 & 117.738 & 58.05 \\
\hline $\begin{array}{l}\text { Swarm } \\
\text { with } \\
\text { linear } \\
\text { threshold }\end{array}$ & 291.52 & 126.73 & 57.85 \\
\hline $\begin{array}{l}\text { Swarm } \\
\text { with ex- } \\
\text { ponential } \\
\text { threshold }\end{array}$ & 299.37 & 120.07 & 56.49 \\
\hline & & & \\
\hline & & & \\
\hline
\end{tabular}

In each case, we also evaluate and compare the different possibilities of our extension of the specialization model (the success function), as defined in Section III-D.Thus, our approach was simulated in three different ways:

- using the original idea about the threshold, updating the threshold with a learning and forgetting coefficients, Equation with a fix value for $l$.

- using the linear function to update the threshold, Equation 7 ;

- using the exponential function to update the threshold, Equation 8;

In this paper we adopt $\alpha=0.2, \beta=0.5$ and $\theta$ starting with 0.5 . When changing the threshold using the original idea we adopt $\xi=0.5$ and $\rho=0.05$. Our extension uses the linear function presented in Equation 7 and the exponential function presented in Equation 8.

Table I shows values of average travel times for the FCs, collected over three hour of simulation (10800 simulation steps). The travel times were measured with three FCs in each route (arterial, secondary 1 and 2). In the table we give average travel times over the three FCs (in the same route) and over the whole simulation time. The time to complete a entire route without having delays caused by traffic lights (free flow) is shown in the first row. This time is shown here only for sake of comparison (a lower bound); this situation could never occur in real traffic networks since the times were obtained simulating a situation in which vehicles do not stop (or even reduce speed) at crossings.

A situation more close to the actual one in this part of the network in Porto Alegre is shown in the third line of the table: no synchronization. In this case, the travel times of the FCs (in each route) are much higher because they stop very often. 
When only a fixed coordination in the arterial is used, drivers in the arterial are of course better off. However, this harms the traffic flow conditions in the two secondary streets. In fact, for the Secondary 1 this is the worst situation since the FC takes 288 seconds to complete the route (compared to 37 in free flow!). Therefore, as seen in Section I, this coordination can be inefficient if the traffic volume in the arterial is low. For instance, if the traffic flow in the secondary streets increases while the arterial stays almost empty, only a adapting approach can deal with this. Our approach is able to perceive this difference and to adapt the traffic lights to priorize the higher traffic flow.

The swarm coordination approach, using any threshold update model, gives priority to the street with the worst traffic conditions (in this case, the 2nd secondary street). The three different threshold update models have a very similar behavior in terms of priorization, this occurs mainly due the lack of change $n$ the traffic scenario, since the traffic lights have almost always the same stimulus for each plan. If the traffic conditions changes, the threshold model could have an impact in the change of tasks (plans).

\section{Conclusions and Outlook}

This paper proposes an approach to reduce traffic jams based on a swarm-inspired method of selecting signal plans. Some approaches were presented to reduce traffic jams, focusing on signal plan selection, either via classical approaches or via more flexible ones like the one proposed in [1]. There is a clear need for even more flexible approaches in which the preferences of the traffic lights regarding the coordination or synchronization do not have to be explicitly stated.

The swarm approach is well suited here because it profits from the metaphor of vehicles leaving a pheromone trail when stopped at a junction. This metaphor is used as a kind of stigmergy between adjacent junctions.

The approach was realized in a microscopic traffic simulator, to which models of social insects were added. These insects thus perceive the pheromone trails and act accordingly which in this case means a selection of an appropriate signal plan.

The average time to complete a route in three different streets was measured in order to compare the following situations: i) the traffic lights are not coordinated; ii) they are coordinated in the classical way, i.e., using a central decision component (normally the traffic engineer) which determines the unique synchronization for all junctions; iii) they are free to decide, at local level, whether or not to coordinate. This last approach is more flexible and depends only on detectors installed at each junction.

Quantitatively, when the agents are free to decide coordinating according to the swarm approach, the system behaves almost as if a central decision support was given. Our experiments shows that the agents achieve synchronization without any management, that indicates a successful swarm based application.

The present work foresees some extensions as for instance increasing the set of signal plans an insect "controls". Additional signal plans can be designed either to coordinate in other directions or to coordinate in the main direction with other shares of green time and offsets. To implement this, we depend on the traffic engineer who has to design such plans.

Other possible extensions are the simulation of the extended network (which is currently being done and again, depends on the engineers) so to consider parallel streets and so on.

\section{ACKNOWLEDGEMENTS}

Denise de Oliveira and Ana L. C. Bazzan are partially supported by CAPES and CNPq respectively. This work was developed with support of CAPES (with the state of Bavaria and DAAD - PROBRAL), as well as of BIC/FAPERGS.

\section{Author Biographies}

DENISE DE OLIVEIRA is a PhD student at the Univ. of Rio Grande do Sul (UFRGS), Brazil, Computer Science Dept. Her main interests are in agent-based simulation, reinforcement learning, Markov decision processes, and coordination in multiagent systems.

ANA L. C. BAZZAN is an adjunct professor at the Univ. of Rio Grande do Sul (UFRGS), Brazil, Computer Science Dept. She has a PhD from the Univ. of Karlsruhe, Germany, with a thesis on an Evolutionary Game-Theoretic Approach to Coordination of Traffic Signal Agents. Currently she coordinates a project on Intelligent Traffic Systems, and is a member of the board of the Int. Foundation of Autonomous Agents and Multiagent Systems (IFAAMAS), as well as of several program committees.

\section{REFERENCES}

[1] Ana L. C. Bazzan. A distributed approach for coordination of traffic signal agents. Autonomous Agents and Multiagent Systems, 10(1):131-164, March 2005.

[2] E. Bonabeau, G. Thraulaz, and M. Dorigo. Swarm Intelligence: From Natural to Artificial Systems. Oxford Univ Press, 1999.

[3] D. Gordon. The organization of work in social insect colonies. Nature, 380:121-124, 1996.

[4] D. Gordon. Ants at Work: How an Insect Society is Organized. W.W. Norton \& Company, 2000.

[5] P. B. Hunt, D. I. Robertson, R. D. Bretherton, and R. I. Winton. SCOOT - a traffic responsive method of coordinating signals. TRRL Lab. Report 1014, Transport and Road Research Laboratory, Berkshire, 1981.

[6] P. Lowrie. The sydney coordinate adaptive traffic system - principles, methodology, algorithms. In Proceedings of the International Conference on Road Traffic Signalling, Sydney, Australia, 1982.

[7] K. Nagel and M. Schreckenberg. A cellular automaton model for freeway traffic. Journal de Physique I, 2:2221, 1992.

[8] Gene E. Robison. Regulation of division of labor in insect societies. Annual Review of Entomology, 37:637-665, 1992.

[9] B. C. Silva, A. L. C. Bazzan, G. K. Andriotti, and D. Oliveira. ITSUMO: an intelligent transportation system for urban mobility. In Proceedings of the Optimization of Urban Traffic Systems, Lecture Notes in Computer Science - Innovative Internet Computing Systems 2004, pages 224-235, Guadalajara, Mexico, 2005. Berlin, Springer-Verlag.

[10] TRANSYT-7F. TRANSYT-7F User's Manual. Transportation Research Center, University of Florida, 1988. 